\title{
A Necessary Condition for the Stability of a Difference Approximation to a Hyperbolic System of Partial Differential Equations
}

\author{
By Anne M. Burns
}

Abstract. We are interested in the boundary conditions for a difference approximation to a hyperbolic system of partial differential equations $u_{t}=A u_{x}, u(x, 0)=$ $F(x), K u(0, t)=0$ in the quarter plane $x \geqslant 0, t \geqslant 0$.

We consider approximations of the type:

$$
\begin{gathered}
u_{j}(t+\Delta t)=\sum_{k=-r}^{p} C_{k} u_{j+k}(t), \quad j=1,2, \ldots, \\
u_{j}+\sum_{k=1}^{s} \alpha_{j k} u_{k}(t+\Delta t)=0, \quad j=-r+1, \ldots, 0 .
\end{gathered}
$$

If $N$ is the null space of $K$ and $E$ is the "negative" eigenspace of $A$, then the system of partial differential equations is well-posed if and only if $K \cap E=\{0\}$ and $\operatorname{Rank} K=$ the number of negative eigenvalues of $A$.

In direct analogy to this, we prove that for a difference scheme of the above type with $r=p=1, K^{\prime}=I+\Sigma_{k=1}^{s} \alpha_{k}$ and $N^{\prime}=$ null space of $K^{\prime}$, a necessary condition for stability is $N^{\prime} \cap E=\{0\}$. If, in addition, a condition proven by S. J. Osher to be sufficient for stability is not satisfied, then Rank $K=$ the number of negative eigenvalues of $A$ is also necessary for stability. We then generalize this result to the case $r>1, p>1$.

Together these conditions imply that "extrapolation" on "negative" eigenvectors leads to instability; "extrapolation" on "positive" eigenvectors is "almost necessary. "Extrapolation" on "positive" eigenvectors and not on "negative" eigenvectors is sufficient for stability.

1. Introduction. Here we consider the problem of finding the correct boundary conditions for a difference approximation to the hyperbolic system of partial differential equations:

$$
\begin{aligned}
u_{t} & =A u_{x}, \\
u(x, 0) & =F(x), \\
K u(0, t) & =G(t),
\end{aligned}
$$

in the quarter plane $x \geqslant 0, t \geqslant 0$, where $A$ is a constant, symmetric $n \times n$ matrix with eigenvalues $a_{1}<\cdots<a_{m}<0<a_{m+1}<\cdots<a_{n}$ and $K$ is a constant $n \times n$ matrix. 
A necessary and sufficient condition for the problem to be well-posed is

(i) Rank $K=m=$ the number of negative eigenvalues of $A$, and

(ii) $N \cap E=\{0\}$ where $N$ is the null space of $K$ and $E$ is the "negative" eigenspace of $A$, that is, the $m$-dimensional subspace of $\mathbf{C}^{n}$ spanned by the eigenvectors of $A$ which correspond to the negative eigenvalues of $A$. (See Hersh [2] .)

Introduce a mesh width $\Delta x>0$ and a time step $\Delta t>0$; divide the $x$-axis into subintervals of length $\Delta x$, the $t$-axis into subintervals of length $\Delta t$, and assume the ratio $r=\Delta t / \Delta x$ remains constant.

Let $u_{j}(t)=u(j \Delta x, t)$ be an $n \times 1$ vector function of $x$ and $t$ :

$$
u_{j}(t)=\left(u_{j}^{(1)}(t), \ldots, u_{j}^{(n)}(t)\right)^{t}
$$

For simplicity, we begin by considering a particular class of difference approximations to (1.1):

$$
\begin{gathered}
u_{j}(t+\Delta t)=\sum_{k=-1}^{1} C_{k} u_{j+k}(t), \quad j=1,2, \ldots, \\
u_{j}(0)=f_{j},
\end{gathered}
$$

where the $C_{k}$ 's are constant $n \times n$ matrices which depend on $A$ and $\Delta t$. For example, the Lax-Wendroff Scheme:

$$
\begin{aligned}
u_{j}(t+\Delta t)= & u_{j}(t)+\frac{1}{2} \frac{\Delta t}{\Delta x} A\left(u_{j+1}(t)-u_{j-1}(t)\right) \\
& +\frac{1}{2}\left(\frac{\Delta t}{\Delta x}\right)^{2} A^{2}\left(u_{j+1}(t)-2 u_{j}(t)+u_{j-1}(t)\right) .
\end{aligned}
$$

Even in this case where there is only one backward step, we see that in contrast to the partial differential equation where $m$ boundary conditions are required, here to solve the difference scheme at each time step, we require $n$ boundary conditions. They will be given by:

$$
\sum_{k=0}^{s} \alpha_{k} u_{k}(t+\Delta t)=0
$$

where the $\alpha_{k}$ 's are constant $n \times n$ matrices and $\alpha_{0}=I$.

The problem, then, is what are the proper extra $n-m$ boundary conditions.

The requirement of consistency suggests using extrapolation at the boundary: using a Taylor series expansion in powers of $\Delta x$ to determine the $\alpha_{k}$ 's. (Shortly, we shall be more precise.)

Let $K^{\prime}=\Sigma_{k=0}^{s} \alpha_{k}$ and $E$ be the "negative" eigenspace of $A$.

Then in this paper we shall show that a necessary condition for stability is $K^{\prime} \cap E=\{0\}$. If, in addition, a certain algebraic condition shown by Osher in [6] and Kreiss in [3] is not satisfied, then it is also necessary that Rank $K^{\prime}=m=$ the number of negative eigenvalues of $A$. We shall show how this relates to extrapolating at the boundary and how the result is extended to a more general class of difference schemes. 
2. Some Preliminary Notions. Let $H$ be the Hilbert space of sequences $w=$ $\left\{w_{j}\right\}, w_{j}=\left(w_{j}^{(1)}, \ldots, w_{j}^{(n)}\right)^{t}, j=0,1,2, \ldots$, such that $\Sigma_{j=0}^{\infty}\left|w_{j}\right|^{2}<\infty$ where $w_{j} \cdot v_{j}=\Sigma_{i=1}^{n} w_{j}^{(i)} \bar{v}_{j}^{(i)}\left|w_{j}\right|^{2}=w_{j} \cdot w_{j}$, with scalar product $(w, v)=\left(\sum_{j=0}^{\infty} w_{j} \cdot v_{j}\right) \Delta x$ and norm $\|w\|^{2}=(w, w)$.

Let $S$ be the operator on $H$ defined by

$$
\begin{aligned}
(S w)_{j} & =\sum_{k=-1}^{1} C_{k} w_{j+k}, \quad j=1,2, \ldots, \\
(S w)_{0} & =-\sum_{k=1}^{s} \alpha_{k}(S w)_{k} .
\end{aligned}
$$

Then we can write the difference approximation (1.4), (1.5), (1.6) in operator form:

$$
u(t+\Delta t)=(S u)(t)=\left(S^{n} u\right)(0) \text {. }
$$

Let $v(x, t)$ be the genuine solution to the differential equation (1.1), (1.2), (1.3) for some smooth initial function $f$, where $\widetilde{f}=\{f(j \Delta x)\} \in H$. Let $\widetilde{v}(t)=$ $\left\{\widetilde{v}_{j}(t)\right\}=\{v(j \Delta x, t)\}$. Then $\tilde{v}(t) \in H$.

By convergence of the difference scheme to the solution of the partial differential equation we mean:

There exists a sequence $\Delta_{j} t \rightarrow 0$ and a sequence of integers $n_{j}$ such that $n_{j} \Delta_{j} t$ $\rightarrow t$ as $j \rightarrow \infty$ and $\left\|S^{n_{j}}\left(\Delta_{j} t\right) \tilde{v}(0)-\tilde{v}(t)\right\| \longrightarrow 0$ as $j \rightarrow \infty$.

(2.1) Definition. The difference scheme is a consistent approximation to the differential equation if for any smooth solution $v(x, t)$ of $(1.1),(1.2),(1.3)$

$$
\left\|\frac{\tilde{v}(t+\Delta t)-(\tilde{S} \tilde{v})(t)}{\Delta t}\right\| \rightarrow 0 \text { as } \Delta t \rightarrow 0 \text { for } t \leqslant T .
$$

(2.2) Definition. The difference scheme (1.4), (1.5), (1.6) is stable if there exists $\delta>0$ and a constant $k>0$ such that $\left\|S^{m}\right\| \leqslant k$ when $0<\Delta t<\delta$ and $0 \leqslant$ $m \Delta t \leqslant T$.

Then the well-known Lax-Richtmyer Theorem states that for a consistent difference approximation stability is equivalent to convergence.

Now we are in a position to see why consistency suggests extrapolating at the boundary.

Consistency (2.1) implies that for all $j$ and any genuine solution of the partial differential equation:

$$
\left|\frac{\tilde{u}_{j}(t+\Delta t)-(S \tilde{u})_{j}(t)}{\Delta t}\right| \rightarrow 0
$$

In particular,

$$
\sum_{k=0}^{s} \alpha_{k} u_{k}(t+\Delta t)=O\left((\Delta t)^{2}\right)=O\left((\Delta x)^{2}\right) .
$$

For example, in the scalar case with $s=2$ : 


$$
\begin{aligned}
u_{0}+\alpha_{1} u_{1}+\alpha_{2} u_{2} & =u_{0}+\alpha_{1}\left(u_{0}+\Delta x\left(u_{x}\right)_{0}\right)+\alpha_{2}\left(u_{0}+(2 \Delta x)\left(u_{x}\right)_{0}\right) \\
& =O\left((\Delta x)^{2}\right)
\end{aligned}
$$

yields $u_{0}-2 u_{1}+u_{2}=0$.

An important fact which will be used later is: if the boundary conditions $\Sigma_{k=0}^{s} \alpha_{k} u_{k}=O\left((\Delta x)^{s}\right)$, then $\mu=1$ is a root of the polynomial $\Sigma_{k=0}^{s} \alpha_{k} \mu^{k}=0$ of multiplicity $s$.

Now consider the matrix case, $n \geqslant 2$. We shall say we are extrapolating on the vector $\phi$ if the $\alpha_{k}$ 's satisfy $\Sigma_{k=0}^{s} \alpha_{k} w(k \Delta x) \phi=O\left((\Delta x)^{s}\right)$ for a sufficiently differentiable scalar function $w(x)$. In the following example we extrapolate on the vector $(1,0)^{t}$ :

$$
u_{0}+\left[\begin{array}{cc}
-2 & 0 \\
k_{1} & k_{2}
\end{array}\right] u_{1}+\left[\begin{array}{cc}
1 & 0 \\
k_{3} & k_{4}
\end{array}\right] u_{2}=0
$$

Extrapolating on a vector $\phi$ implies that $K^{\prime} \phi=\Sigma_{k=0}^{s} \alpha_{k} \phi=0$.

We now restate our main result: Under certain assumptions which are necessary for the stability of the Cauchy problem, if $\phi \in E$, then a necessary condition for stability is $\left(\Sigma_{k=0}^{s} \alpha_{k}\right) \phi \neq 0$ or equivalently $N^{\prime} \cap E=\{0\}$, where $N^{\prime}$ is the null space of $K^{\prime}=\Sigma_{k=0}^{s} \alpha_{k}$.

This gives us rank $K \geqslant m$. If, in addition, we assume that $S$ has an eigenvalue $z=1$, then it is also necessary that $\operatorname{rank} K=m$.

In other words we cannot extrapolate on the "negative" eigenvectors of $A$. In fact, we shall show that the higher the order of extrapolation on negative eigenvectors of $A$ the more unstable the scheme. If $S$ has an eigenvalue at $z=1$, we obtain instability unless we do extrapolate on the "positive" eigenvectors of $A$.

3. The (Generalized) Eigenvalues of $S$. In the proof of the Main Theorem we require some knowledge about the eigenvalues of $S$.

In addition to the consistency of the scheme we make the following assumptions:

(3.1) The Cauchy problem is stable. In particular, this implies the von Neumann condition: for $|\mu|=1$ the eigenvalues $z$ of $\Sigma_{k=-1}^{1} C_{k} \mu^{k}$ satisfy $|z| \leqslant 1$.

(3.2) The $C_{k}$ 's are polynomials in $A$ with coefficients which are functions of $\Delta t$. (See again the Lax-Wendroff scheme:) This implies that the eigenvectors of $\Sigma_{k=-1}^{1} C_{k} \mu^{k}$ are the eigenvectors of $A$, for $\mu \in \mathbf{C}$.

If $z$ is an eigenvalue of $S$, then there exists $u \in H$ such that

$$
\begin{gathered}
\sum_{k=-1}^{1} C_{k} u_{j+k}-z u_{j}=0, \quad j=1,2, \ldots, \\
z u_{0}=-\sum_{k=1}^{s} \alpha_{k}(S u)_{k} .
\end{gathered}
$$

It is well known that the general solution in $H$ to (3.3) for $|z|>1$ is 


$$
u_{j}=u_{j}(z)=\sum_{\left|\mu_{i}\right|<1} \sigma_{i} \mu_{i}^{j}(z) \phi_{i},
$$

where $\mu_{i}(z)$ are the solutions to

$$
\operatorname{Det}\left(\sum_{k=-1}^{1} C_{k} \mu^{k}-z I\right)=0,
$$

which lie inside the unit circle and the $\phi_{i}$ satisfy

$$
\sum_{k=-1}^{1} C_{k} \mu_{i}^{k} \phi_{i}=z \phi_{i}
$$

By (3.2) the $\phi_{i}$ must be eigenvectors of $A$. For convenience wo make the following assumption

$$
C_{1} \text { and } C_{-1} \text { are nonsingular (see Lax-Wendroff). }
$$

LEMMA 1. Under assumptions (3.1), (3.2), (3.7) and consistency:

(a) For $|z|>1$ there are $2 n$ solutions $\mu(z)$ to (3.5); $n$ of them satisfy $|\mu(z)|$ $<1$ and $n$ of them satisfy $|\mu(z)|>1$. They are analytic functions of $z$ except possibly at branch points.

(b) Corresponding to each eigenvalue of $A, a_{i}$, two of these solutions satisfy (3.5): $\mu_{i 1}(z)$ which satisfies $\left|\mu_{i 1}(z)\right|<1$ for $|z|>1$ and $\mu_{i 2}(z)$ which satisfies $\left|\mu_{i 2}(z)\right|>1$ for $|z|>1$. As $z \rightarrow 1$ precisely one of these approaches 1 ; if $i=1$, $\ldots, m, \mu_{i 1}(z) \rightarrow 1$ as $z \rightarrow 1$; if $i=m+1, \ldots, n, \mu_{i 2}(z) \rightarrow 1$ as $z \rightarrow 1$.

Proof of (a). Let

$$
\begin{aligned}
f(\mu, z) & =\operatorname{Det}\left(\sum_{k=-1}^{1} C_{k} \mu^{k}-z I\right) \\
& =\left(\operatorname{Det} C_{-1}\right) \mu^{-n}+\cdots+\left(\operatorname{Det} C_{1}\right) \mu^{n} \\
& =\mu^{-n}\left[\left(\operatorname{Det} C_{-1}\right)+\cdots+\left(\operatorname{Det} C_{1}\right) \mu^{2 n}\right]
\end{aligned}
$$

Fix $\left|z_{0}\right|>1$. Then by (3.7) $f\left(\mu, z_{0}\right)$ has $2 n$ zeros $\mu\left(z_{0}\right) \neq 0$. Each $\mu(z)$ is analytic in $z$ except perhaps at branch points.

We may also write

$$
f(\mu, z)=\prod_{i=1}^{n}\left(z_{i}(\mu)-z\right),
$$

where the $z_{i}(\mu)$ are the eigenvalues of $\Sigma_{k=-1}^{1} C_{k} \mu^{k}$ (which is diagonalizable since it has a spanning set of eigenvectors by (3.2)).

Since $\left|z_{i}(\mu)\right| \leqslant 1$ for $|\mu|=1$ by (3.1), we may take $\left|z_{0}\right|$ sufficiently large so that

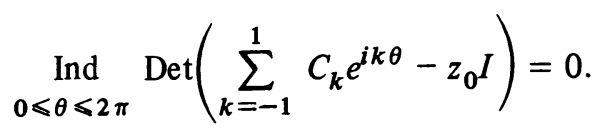

Therefore, the number of zeros $\mu\left(z_{0}\right)$ of $f\left(\mu, z_{0}\right)$ inside the unit circle equals the number of poles of $f\left(\mu, z_{0}\right)$ at 0 which is $n$ (from representation (3.8) of $f\left(\mu, z_{0}\right)$ and 
assumption (3.7)). Similarly, the number of zeros of $f\left(\mu, z_{0}\right)$ outside the unit circle equals the number of poles at $\infty$; this number is also $n$ by (3.8) and (3.7).

Now, if we let $z$ vary outside the unit circle, the functions $\mu(z)$ cannot cross over the unit circle since by (3.1) if $|\mu|=1$, then $|z| \leqslant 1$.

Proof of (b). Suppose $\operatorname{Det}\left(\Sigma_{k=-1}^{1} C_{k} \mu^{k}-z I\right)=0$. Then there exists $\phi$ such that $\Sigma_{k=-1}^{1} C_{k} \mu^{k} \phi=z \phi$ and $\phi=\phi_{i}$, one of the eigenvectors of $A$.

Then

$$
z \phi_{i}=\sum_{k=-1}^{1} C_{k} \mu^{k} \phi_{i}=g_{i}(\mu) \phi_{i} \quad \text { or } \quad z=g_{i}(\mu),
$$

$g_{i}(\mu)$ must be of the form $a_{-1}^{(i)} \mu^{-1}+a_{0}^{(i)}+a_{1}^{(i)} \mu$; and thus, there can be no more than two roots $\mu(z)$ of $g_{i}(\mu)-z=0$. Since (3.5) has $2 n$ roots, it follows that for each $i$ there are two roots $\mu(z)$ satisfying (3.6).

By consistency we can write

$$
(z-1) \phi_{i}=\left\{\frac{\Delta t}{\Delta x} A(\mu-1)+(\mu-1)^{2} P(\mu-1)\right\} \phi_{i}, \quad i=1, \ldots, n,
$$

or

$$
z-1=\frac{\Delta t}{\Delta x} a_{i}(\mu-1)+(\mu-1)^{2} p(\mu-1)
$$

where $p$ is an infinite series in powers of $\mu-1$ convergent near $\mu=1$. Thus, for each $i$ exactly one of the two roots $\mu_{i}(z)$ approaches 1 as $z \rightarrow 1$.

Also, this implies that we can choose $z$ real and close to 1 so that $\left|(\mu-1)^{2} p(\mu-1)\right|$ $\langle|z-1|$ for this value of $\mu$.

Then if we rewrite the above as

$$
\mu=1+\frac{z-1}{\Delta t a_{i} / \Delta x}-\frac{(\mu-1)^{2} p(\mu-1)}{\Delta t a_{i} / \Delta x},
$$

we see for $z>1$ and $a_{i}>0,|\mu|>1$, for $z>1$ and $a_{i}<0,|\mu|<1$.

For $i=1, \ldots, n$ and $|z|>1$ we shall denote by $\mu_{i 1}(z)$ the solution to (3.5), which is inside the circle and by $\mu_{i 2}(z)$ the solution to (3.5) which is outside the unit circle. Then, for $i=1, \ldots, m, \mu_{i 1}(1)=1$ and $\mu_{i 2}(1) \neq 1$ and for $i=m+1, \ldots$, $n, \mu_{i 2}(1)=1$ and $\mu_{i 1}(1) \neq 1$.

It follows from the proof of part (b) that for $i=1, \ldots, n$, we can write

$$
z-1=d_{i}\left(1-\frac{\mu_{i 1}(1)}{\mu}\right)\left(\mu-\mu_{i 2}(1)\right), d_{i} \text { constant; }
$$

and therefore, we have

CoROllary. For $v=1,2, \mu_{i v}(z)-\mu_{i v}(1)=(z-1) f_{i v}(z)$, where $0<k_{1} \leqslant$ $\left|f_{i v}(z)\right| \leqslant k_{2}$ for $|z|>1$ and $z$ sufficiently near 1 .

We shall also require

LEMMA 2. The zeros $\mu_{i 2}(z)$ of (3.5) are real if $z$ is real and $z>1$.

Proof. If $z$ and $\mu$ satisfy (3.5), then whenever $\mu$ is real, $z$ is real by (3.2).

Let $\mu$ be a zero of (3.5) which is outside the unit circle for $|z|>1$. Then $\mu$ 
satisfies

$$
z-1=d_{i}\left(1-\frac{\mu_{i 1}(1)}{\mu}\right)(\mu-1), \quad \text { where } i=m+1, \ldots, n ;
$$

and hence $\mu_{i 1}(1)=1$. Since $z$ is real whenever $\mu$ is real, $d_{i}$ and $\mu_{i}(1)$ are real.

Now assume $z$ is real and $z>1$; then $\mu-\left(\mu_{i 1}(1)+1\right)+\mu_{i 1}(1) / \mu$ is real; and thus, $\operatorname{Im} \mu=-\mu_{i 1}(1) \operatorname{Im} \mu^{-1}$, where $-1 \leqslant \mu_{i 1}(1)<1$, which is impossible unless $\operatorname{Im} \mu=\operatorname{Im} \mu^{-1}=0$.

The case $|\mu|<1$ now follows easily from this.

Now we have: the general solution in $H$ to the eigenvector problem (3.3) is $u(z)$, where

$$
u_{j}=u_{j}(z)=\sum_{i=1}^{n} \sigma_{i} \mu_{i 1}^{j}(z) \phi_{i}
$$

Putting this into the boundary condition (3.4), we obtain

$$
\sum_{i=1}^{n} \sigma_{i}\left(\sum_{k=0}^{s} \alpha_{k} \mu^{k}\right) \phi_{i}=0
$$

Then, (3.9) will be an eigenvector of $S$ with corresponding eigenvalue $z,|z|>1$, if and only if there exist $\sigma_{1}, \ldots, \sigma_{n}$ not all zero such that (3.10) holds.

Now from Lemma 1 , as $z \rightarrow 1, \mu_{i 1}(z) \rightarrow 1$ for $i=1, \ldots, m$, so that

$$
u_{j}(1)=\sum_{i=1}^{n} \sigma_{i} \mu_{i 1}^{j}(1) \phi_{i}
$$

will not in general belong to $H$.

Definition. $z=1$ is a generalized eigenvalue of $S$ if (3.10) has a nontrivial solution $\sigma_{1}, \ldots, \sigma_{n}$ for $z=1$. The corresponding solution (3.11) is called a generalized eigenfunction.

In [6] Osher proves that for diagonal $A, S$ has no generalized eigenvalue $z$ with $|z|=1$ is sufficient for stability. Kreiss in [3] under the additional assumption of dissipativity shows that $S$ has no generalized eigenvalue $z$ with $z=1$ implies stability.

4. A Necessary Condition for Stability-Main Theorem. Recall that $E$ is the subspace of $\mathbf{C}^{n}$ spanned by the "negative" eigenvectors of $A$,

$$
K^{\prime}=\sum_{k=0}^{s} \alpha_{k} \quad \text { and } \quad N^{\prime}=\text { null space of } K^{\prime} .
$$

MAIN THEOREM. A necessary condition for the stability of the difference scheme (1.4), (1.5), (1.6) is

$$
N^{\prime} \cap E=\{0\} .
$$

If, in addition, 1 is a generalized eigenvalue of $S$, then Rank $K^{\prime}=m$ is also necessary for stability.

Notice that if we extrapolate on a "negative" eigenvector $\phi_{i}$ of $A$, then 


$$
0=\sum_{k=0}^{s} \alpha_{k} \phi_{i}=\sum_{k=0}^{s} \alpha_{k} \mu_{i 1}^{k}(1) \phi_{i}
$$

Thus, the theorem implies that we cannot extrapolate on "negative" eigenvectors of $A$. If we extrapolate only on "positive" eigenvectors of $A$, then since $\mu_{i 2}(1) \neq 1$ for $i=m+1, \ldots, n, S$ will have no generalized eigenvalue $z=1$. Also, Rank $K^{\prime}=m$ implies extrapolation on "positive" eigenvalues.

Proof of Theorem (1st assertion). Assume that $N^{\prime} \cap E \neq\{0\}$. Then there exist $\sigma_{1}, \ldots, \sigma_{n}$ such that

$$
\sum_{k=0}^{s} \alpha_{k}\left(\sum_{i=1}^{n} \sigma_{i} \mu_{i 1}^{k}(1) \phi_{i}\right)=0
$$

and $\sigma_{i_{0}} \neq 0$ for some $i_{0}, 1 \leqslant i_{0} \leqslant m$. Let $u(z) \in H$ be defined by

$$
u_{j}(z)=\sum_{i=1}^{n} \sigma_{i} \mu_{i 1}^{j}(z) \phi_{i} \quad \text { for } z>1
$$

(in all that follows it suffices to consider real $z$ ).

Since $A$ is symmetric, we may choose $\left\{\phi_{i}\right\}$ to be orthonormal. Then

$$
\begin{aligned}
\|u(z)\| & =\left[\sum_{j=0}^{\infty}\left|\sum_{i=1}^{n} \sigma_{i} \mu_{i 1}^{j}(z) \phi_{i}\right|^{2} \Delta x\right]^{1 / 2} \\
& \geqslant\left[\sum_{j=0}^{\infty}\left|\sigma_{i_{0}}\right|^{2}\left|\mu_{i_{0}}(z)\right|^{2 j} \Delta x\right]^{1 / 2} \\
& =\frac{\left|\sigma_{i_{0}}\right|(\Delta x)^{1 / 2}}{\left(1-\left|\mu_{i_{0}}(z)\right|\right)^{1 / 2}\left(1+\left|\mu_{i_{0}}(z)\right|\right)^{1 / 2}} \\
& =g(z)(z-1)^{-1 / 2}, \text { where }|g(z)| \geqslant k_{1}>0
\end{aligned}
$$

for $z$ sufficiently near 1 by Lemma 2 . Then for $z>1, j=1,2, \ldots,[(S-z) u(z)]_{j}$ $=0$ and

$$
\begin{aligned}
{[(S-z) u]_{0} } & =-z \sum_{k=0}^{s} \alpha_{k}\left(\sum_{i=1}^{n} \sigma_{i 1}^{k}(z) \phi_{i}\right) \\
& =z \sum_{k=0}^{s} \alpha_{k}\left(\sum_{i=1}^{n} \sigma_{i}\left(\mu_{i 1}^{k}(1)-\mu_{i 1}^{k}(z)\right) \phi_{i}\right) \\
& =z \sum_{k=0}^{s} \alpha_{k}\left(\sum_{i=1}^{n} \sigma_{i}(z-1) f_{i k}(z) \phi_{i}\right)
\end{aligned}
$$

where $\left|f_{i k}(z)\right| \leqslant k_{2}$ for $z>1$, by the Corollary to Lemma 1. Hence, $\|(S-z) u(z)\|$ $=(z-1) f(z)$, where $f(z) \leqslant k_{3}$ for $z>1$ and $z$ near 1 .

LEMMA 3. If $\left\|S^{m}\right\| \leqslant k$ for all $0<\Delta t<\delta, 0 \leqslant m \Delta t \leqslant T$, then for all $z$ with $|z|>1,\left\|(S-z)^{-1}\right\| \leqslant k /(|z|-1)$.

Proof. It is obvious that $\left\|S^{m}\right\| \leqslant k$ implies that the spectrum of $S$ is contained 
in the unit disc. Then for $|z|>1$,

$$
(S-z)^{-1}=-\sum_{m=0}^{\infty} z^{-m-1} S^{m} .
$$

Therefore,

$$
\left\|(S-z)^{-1}\right\| \leqslant \sum_{m=0}^{\infty}|z|^{-m-1}\left\|S^{m}\right\| \leqslant \frac{k}{|z|-1} .
$$

Now from above we have for $z>1$ and $z$ near 1 :

$$
\left\|(S-z)^{-1}\right\| \geqslant \frac{\|u(z)\|}{\|(S-z) u(z)\|}=\frac{(z-1)^{-1 / 2} g(z)}{(z-1) f(z)},
$$

$g(z) \geqslant k_{1}>0$ and $f(z) \leqslant k_{3}$. Thus, $\left\|(S-z)^{-1}\right\| \geqslant(z-1)^{-3 / 2} k_{4}$, where $k_{4}>0$ for $z>1$ and $z$ near 1 , violating Lemma 3 and implying the instability of the scheme.

We have now shown that $E \cap N^{\prime}=\{0\}$ is necessary, and thus rank $K^{\prime} \geqslant m$ is also necessary.

To prove the second assertion in the theorem, we assume $S$ has a generalized eigenvalue $z=1$. Then there exist $\sigma_{1}, \ldots, \sigma_{n}$ not all zero such that

$$
\sum_{k=0}^{s} \alpha_{k}\left(\sum_{i=1}^{n} \sigma_{i} \mu_{i 1}^{k}(1) \phi_{i}\right)=0 .
$$

We have just shown that if the scheme is stable, then $\sigma_{i}=0$ for $i=1, \ldots$, $m$. Suppose now that $\sigma_{i}=0$ for $i=1, \ldots, m$ but $\sigma_{i} \neq 0$ for at least one $i=m+$ $1, \ldots, n$.

Two cases can occur.

First: $N^{\prime}=E^{\perp}$ and rank $K^{\prime}=m$, which proves the second assertion in the theorem.

Thus, we shall assume the second case: there exist $\phi_{i}, i=m+1, \ldots, n$, such that

$$
\sum_{k=0}^{s} \alpha_{k} \phi_{i} \neq 0
$$

To show instability in this case we explicitly compute the resolvent $(S-z)^{-1}$. Again, we shall show that under this assumption the resolvent grows too rapidly as $z \rightarrow 1$.

To simplify the calculations we make use of the following

LEMMA 4. Let $H^{\prime}$ be the subspace of $H$ of sequences satisfying the boundary conditions. That is:

$$
H^{\prime}=\left\{u \in H \mid \sum_{k=0}^{s} \alpha_{k} u_{k}=0\right\} .
$$

If $|z|>1$, then $(S-z) H^{\prime}=H^{\prime}$.

Proof. The inclusion $(S-z) H^{\prime} \subseteq H^{\prime}$ is obvious.

Let $f \in H^{\prime}$. Then $f=(S-z) u, u \in H$, so that 


$$
f_{j}=\sum_{k=-1}^{1} C_{k} u_{j+k}-z u_{j}, \quad j \geqslant 1
$$

and

$$
f_{0}=(S u)_{0}-z u_{0}=-\sum_{k=1}^{s} \alpha_{k}\left(f_{k}+z u_{k}\right)-z u_{0}
$$

Therefore,

$$
z \sum_{k=0}^{s} \alpha_{k} u_{k}=-\sum_{k=0}^{s} \alpha_{k} f_{k}=0
$$

Thus, in computing $f=(S-z)^{-1} v$ for $z>1$, if we agree to take $v \in H^{\prime}$, we may assume $f \in H^{\prime} . f=(S-z)^{-1} v$ is equivalent to $(S-z) f=v$. First, we look for the general solution to

$$
\sum_{k=-1}^{1} C_{k} f_{j+k}-z f_{j}=v_{j}, \quad j=1,2, \ldots
$$

This becomes

$$
f_{j+1}=-C_{1}^{-1}\left(C_{-1} f_{j-1}+\left(C_{0}-z\right) f_{j}-v_{j}\right), \quad j \geqslant 1
$$

Let

$$
y_{j+1}=\left(f_{j}, f_{j-1}\right)_{2 n \times 1}^{t}, \quad y_{1}=\left(f_{0}, 0\right)_{2 n \times 1}^{t},
$$

then $y_{j+1}=M y_{j}+g_{j}$, where

$$
M=\left[\begin{array}{cc}
-C_{1}^{-1}\left(C_{0}-z\right) & -C_{1}^{-1} C_{-1} \\
I & 0
\end{array}\right]_{2 n \times 2 n}, \quad g_{j}=\left[\begin{array}{c}
C_{1}^{-1} v_{j-1} \\
0
\end{array}\right]_{2 n \times 1}
$$

The eigenvalues of $M$ are the solutions $\mu_{i \nu}, i=1, \ldots, n, \nu=1,2$, to (3.5) with corresponding eigenvectors

$$
\left[\begin{array}{c}
\mu_{i \nu} \phi_{i} \\
\phi_{i}
\end{array}\right]
$$

where $\Sigma_{k=-1}^{1} C_{k} \mu_{i \nu}^{k}(z) \phi_{i}=z \phi_{i}$.

LEMMA 5. There exist $n \times n$ matrices $M_{1}(z)$ and $\dot{M}_{2}(z)$, analytic for $|z| \geqslant 1$ such that $M_{1}=M_{1}(z)$ has eigenvalues $\mu_{i 1}(z)$ and eigenvectors $\phi_{i}$ and $M_{2}=M_{2}(z)$ has eigenvalues $\mu_{i 2}(z)$ and eigenvectors $\phi_{i}$.

To see this let $Q$ be the $n \times n$ matrix whose columns are $\phi_{1}, \ldots, \phi_{n}$. Then

$$
M_{\nu}=M_{\nu}(z)=Q\left[\begin{array}{ccc}
\mu_{1 \nu}(z) & & 0 \\
0 & & \mu_{n \nu}(z)
\end{array}\right] Q^{-1} \quad \text { for } \nu=1,2
$$

have the desired properties.

Also, notice that $M_{1}-M_{2}$ is invertible for $|z| \geqslant 1$. Let 


$$
T=T(z)=\left[\begin{array}{cc}
\left(M_{1}-M_{2}\right)^{-1} & -M_{2}\left(M_{1}-M_{2}\right)^{-1} \\
-\left(M_{1}-M_{2}\right)^{-1} & M_{1}\left(M_{1}-M_{2}\right)^{-1}
\end{array}\right]_{2 n \times 2 n}
$$

Then

$$
T^{-1}=T(z)^{-1}=\left[\begin{array}{cc}
M_{1} & M_{2} \\
I & I
\end{array}\right]_{2 n \times 2 n} \text { and } T M T^{-1}=\left[\begin{array}{cc}
M_{1} & 0 \\
0 & M_{2}
\end{array}\right]_{2 n \times 2 n}
$$

(for proof see Appendix 1). Let $w_{j}=T y_{j}$. Then $w_{j}$ is a solution of $w_{j+1}=$ $T M T^{-1} w_{j}+T g_{j}$ or

$$
w_{j+1}=\left[\begin{array}{cc}
M_{1} & 0 \\
0 & M_{2}
\end{array}\right] w_{j}+T g_{j}
$$

Let

$$
w_{j}^{\mathrm{I}}=\left(w_{j}^{(1)}, \ldots, w_{j}^{(n)}\right)^{t}, \quad w_{j}^{\mathrm{II}}=\left(w_{j}^{(n+1)}, \ldots, w_{j}^{(2 n)}\right)^{t}
$$

Then the general solution to $(4.1)$ is

$$
w_{j+1}^{\mathrm{I}}=\sum_{\nu=1}^{j} M_{1}^{j-\nu}\left(T g_{\nu}\right)^{\mathrm{I}}+M_{1}^{j} w_{1}^{\mathrm{I}}, \quad w_{j+1}^{\mathrm{II}}=-\sum_{\nu=j+1}^{\infty} M_{2}^{j-\nu}\left(T g_{\nu}\right)^{\mathrm{II}}
$$

To solve for $w_{1}^{I}$ we use the boundary conditions. We are solving $f=(S-z)^{-1} v$, where both $f$ and $v$ are in $H^{\prime}$. Then $\Sigma_{k=0}^{s} \alpha_{k} f_{k}=0$ becomes

$$
\sum_{k=0}^{s}\left[\begin{array}{ll}
\alpha_{k} & 0 \\
0 & 0
\end{array}\right] y^{k+1}=0
$$

which becomes

$$
\sum_{k=0}^{s}\left[\begin{array}{cc}
\alpha_{k} & 0 \\
0 & 0
\end{array}\right] T^{-1} w_{k+1}=0, \quad \sum_{k=0}^{s}\left[\begin{array}{cc}
\alpha_{k} M_{1} & \alpha_{k} M_{?} \\
0 & 0
\end{array}\right]\left[\begin{array}{l}
w_{k+1}^{I} \\
w_{k+1}^{I I}
\end{array}\right]=0
$$

Putting the general solution (4.2) into this, we obtain:

$$
\begin{aligned}
\sum_{k=0}^{s} \alpha_{k} M_{1}\left(\sum_{\nu=1}^{k} M_{1}^{k-\nu}\left(T g_{\nu}\right)^{\mathrm{I}}+M_{1}^{k} w_{1}^{\mathrm{I}}\right) \\
+\sum_{k=0}^{s} \alpha_{k} M_{2}\left(-\sum_{\nu=k+1}^{\infty} M_{2}^{k-\nu}\left(T g_{\nu}\right)^{\mathrm{II}}\right)=0
\end{aligned}
$$

which becomes

$$
\begin{aligned}
\sum_{k=0}^{s} \alpha_{k} M_{1}^{k+1} w_{1}^{\mathrm{I}}= & -\sum_{k=0}^{s} \alpha_{k} M_{1}\left(\sum_{\nu=1}^{k} M_{1}^{k-\nu}\left(T g_{\nu}\right)^{\mathrm{I}}\right) \\
& +\sum_{k=0}^{s} \alpha_{k}\left(\sum_{\nu=k+1}^{\infty} M_{2}^{k+1-\nu}\left(T g_{\nu}\right)^{\mathrm{II}}\right)
\end{aligned}
$$


Let $C(z)=\Sigma_{k=0}^{s} \alpha_{k} M_{1}^{k+1}$. We shall need

Lemma 6. Det $C(z)=(z-1) f(z)$, where $|f(z)| \leqslant k_{5}$ for $z>1$ and $z$ near 1 ; and hence, $C(z)^{-1}=F(z) /(z-1)$, where

$$
0<k_{6} \leqslant|F(z)|=\sup _{|v|=1}|F(z) v| .
$$

To see this: $S$ has a generalized eigenvalue at $z=1$ implies that Det $C(1)=0$. But Det $C(z)$ is a sum of products of polynomials in $\mu_{i 1}(z), i=1, \ldots, n$, all of degree $\geqslant 1$, and for each $i$ we may write $\mu_{i 1}(z)=\mu_{i 1}(1)+(z-1) f_{i}(z)$, where $f_{i}(1)$ $\neq 0$ from Corollary to Lemma 1 . (For more detailed proof see Appendix 2.)

Now for $z$ real, $z>1$ we choose $x(z) \in H^{\prime}$ so that

$$
\begin{aligned}
& x_{j}(z)=0 \quad \text { for } j=0, \ldots, s, \\
& x_{j}(z)=-C_{1}\left(M_{1}-M_{2}\right) \mu_{i 2}^{s+1-j}(z) \phi_{i}, \quad j=s+1, \ldots,
\end{aligned}
$$

where $\Sigma \alpha_{k} \phi_{i} \neq 0$. By Lemma $2, \mu_{i 2}(z)$ is real and $\mu_{i 2}(z)>1$. This gives

$$
\|x\|=\|x(z)\|=\frac{h_{1}(z)}{(z-1)^{1 / 2}}, \quad \text { where } h_{1}(z) \geqslant k_{6}>0 .
$$

Now let $v_{j}(z)=x_{j}(z) /\|x\|$. Then $\operatorname{Tg}_{j}(z)=0$ for $j=1, \ldots, s+1$ and for $j=s+$ $2, \ldots$,

$$
\left[\begin{array}{c}
\left(T g_{j}\right)^{\mathrm{I}} \\
\left(T g_{j}\right)^{\mathrm{II}}
\end{array}\right]=\left[\begin{array}{c}
\left(T g_{j}(z)\right)^{\mathrm{I}} \\
\left(T g_{j}(z)\right)^{\mathrm{II}}
\end{array}\right]=\left[\begin{array}{c}
\left(M_{1}-M_{2}\right)^{-1} C_{1}^{-1} v_{j-1}(z) \\
-\left(M_{1}-M_{2}\right)^{-1} C_{1}^{-1} v_{j-1}(z)
\end{array}\right]=\left[\begin{array}{c}
\frac{-\mu_{i 2}^{s-j+2}(z) \phi_{i}}{\|x\|} \\
\frac{\mu_{i 2}^{s-j+2}(z) \phi_{i}}{\|x\|}
\end{array}\right] .
$$

Since $T g_{j}=0$ for $j=1, \ldots, s+1$, the first sum on the right-hand side of (4.3) is zero; and (4.3) becomes

$$
\begin{aligned}
C(z) w_{1}^{\mathrm{I}} & =\sum_{k=0}^{s} \alpha_{k}\left(\sum_{j=s+2}^{\infty} M_{2}^{k+1-j}\left(T g_{j}\right)^{\mathrm{II}}\right) \\
& =\frac{1}{\|x\|} \sum_{j=0}^{\infty} \mu_{i 2}^{-2 j}\left(\sum_{k=0}^{s} \alpha_{k} \mu_{i 2}^{k-s-1}(z) \phi_{i}\right) \\
& =(z-1)^{-1 / 2} \frac{h_{1}(z)}{h(z)}\left(\sum_{k=0}^{s} \alpha_{k} \mu_{i 2}^{k-s-1}(z) \phi_{i}\right),
\end{aligned}
$$

where for $z>1$ and sufficiently near 1

$$
h_{1}(z) \geqslant k_{6}>0 \text { and } 0<h(z) \leqslant k_{5} .
$$

We chose $\phi_{i}$ so that $\Sigma_{k=0}^{s} \alpha_{k} \phi_{i} \neq 0$ and since $\mu_{i 2}(z) \rightarrow 1$ as $z \rightarrow 1$

$$
\left|\sum_{k=0}^{s} \alpha_{k} \mu_{i 2}^{k-s-1}(z) \phi_{i}\right| \geqslant k_{7}>0 \text { for } z \text { near } 1 \text {. }
$$


Then

$$
w_{1}^{\mathrm{I}}(z)=(z-1)^{-3 / 2} F(z) \frac{h_{1}(z)}{h(z)}\left(\sum_{k=0}^{s} \alpha_{k} \mu_{i 2}^{k-s-1}(z) \phi_{i}\right),
$$

and hence

$$
\|w(z)\| \geqslant\left|w^{I}(z)\right| \geqslant(z-1)^{-3 / 2} k_{9}, \quad k_{9}>0,
$$

for $z>1$ and $z$ near 1 , so that, as $T^{-1}$ is bounded below for all $z \geqslant 1$,

$$
\left\|(S-z)^{-1} v\right\|=\|f\|=1 / 2\|y\|=1 / 2\left\|T^{-1} w\right\| \geqslant k_{10}\|w\|,
$$

again violating the necessary condition for stability in Lemma 3.

5. Some Remarks and Examples. In the scalar case $u_{t}=a u_{x}$ the general solution to the eigenvalue problem for $|z|>1$ is $u_{j}=\mu^{j}$, where

$$
\sum_{k=-1}^{1} c_{k} \mu^{k}=z, \quad|\mu|<1
$$

Putting this into the boundary conditions, we obtain

$$
\sum_{k=0}^{s} \alpha_{k} \mu^{k}=0, \quad \alpha_{k} \in \mathbf{C}, \alpha_{0}=1
$$

If the boundary conditions are $O\left((\Delta x)^{s}\right)$, then we may write (5.2) as

$$
\sum_{k=0}^{s} \alpha_{k} \mu^{k}=(\mu-1)^{s}
$$

If $a<0$, then $\mu \rightarrow 1$; and as in the proof of the first assertion in the Main Theorem, let $u_{j}(z)=\mu^{j}(z), j=0,1,2, \ldots$, where $(z)$ satisfies $(5.1)$ and $|\mu(z)|<1$.

Then repeating that argument, $\|(S-z) u\|=(z-1)^{s} g(z)$ where $g(z) \leqslant k$ and so $\left\|(S-z)^{-1}\right\| \geqslant k_{1}(z-1)^{-s-1 / 2}$.

Thus, the higher the order of extrapolation on a negative eigenvector, the worse the behavior of $\left\|(S-z)^{-1}\right\|$ as $z \rightarrow 1$.

Example. Consider the Lax-Wendroff approximation to

$$
\begin{aligned}
& u_{t}=\left[\begin{array}{ll}
a_{1} & 0 \\
0 & a_{2}
\end{array}\right] u_{x}, \quad a_{1}<0, a_{2}>0, \\
& {\left[\begin{array}{cc}
1 & -\beta \\
0 & 0
\end{array}\right] u(0, t)=0, \quad 0<|\beta| \leqslant 1 .}
\end{aligned}
$$

The proper boundary conditions for the difference scheme are

$$
u_{0}+\alpha_{1} u_{1}+\alpha_{2} u_{2}=0
$$

where 


$$
\alpha_{1}=\left[\begin{array}{cc}
0 & -2 \beta \\
0 & -2
\end{array}\right], \quad \alpha_{2}=\left[\begin{array}{ll}
0 & \beta \\
0 & 1
\end{array}\right]
$$

This scheme has no generalized eigenvalue at $z=1$ and is stable. If on the other hand, we use the boundary conditions (5.4) with

$$
\alpha_{1}=\left[\begin{array}{cc}
-2 & -2 \beta \\
0 & -2
\end{array}\right], \quad \alpha_{2}=\left[\begin{array}{ll}
1 & \beta \\
0 & 1
\end{array}\right],
$$

then the scheme is unstable since a "negative" eigenvector of $A,(1,0)^{t}$ is in $N^{\prime}$.

If $z=1$ is a generalized eigenvalue of $S$, a necessary condition for stability is $K^{\prime} \phi_{i}=0$ for all $\phi_{i}$ which are "positive" eigenvectors of $A$. Unfortunately, this is not sufficient, for consider again the scalar case $u_{t}=a u_{x}, a>0$, with boundary conditions:

$$
\sum_{k=0}^{s} \alpha_{k} u_{k}(t+\Delta t)=0, \quad \alpha_{0}=1
$$

which satisfy

$$
\sum_{k=0}^{s} \alpha_{k} \mu^{k}=(\mu-1)(\mu-\mu(1))^{s-1}, \quad \text { where } s \geqslant 3 .
$$

This is seen to be unstable by letting $u_{j}(z)=\mu^{j}(z)$ where $\mu$ is the zero of (5.1) which is inside the unit circle for $|z|>1$. In this case $\|u(z)\|$ may remain bounded as $z \rightarrow$ 1 (as in the Lax-Wendroff scheme) but for real $z, z>1$,

$$
\|(S-z) u(z)\|=k(z)(z-1)^{2}, \quad \text { where } k(z) \leqslant k .
$$

Again this leads to

$$
\left\|(S-z)^{-1}\right\| \geqslant k(z-1)^{-2} .
$$

So far we have examined the behavior of the resolvent $(S-z)^{-1}$ only when $S$ has a generalized eigenvalue at $z=1$. For a dissipative scheme the only possible generalized eigenvalue $z$ with $|z|=1$ for $S$ under our assumptions is $z=1$. However, in a nondissipative scheme it is possible for $S$ to have a generalized eigenvalue $z \neq 1$ but $|z|=1$.

For example, consider the leap-frog approximation to the scalar partial differential equation $u_{t}=a u_{x}$ :

$$
\begin{gathered}
u_{j}(t+\Delta t)=u_{j}(t-\Delta t)+\operatorname{ar}\left(u_{j+1}(t)-u_{j-1}(t)\right), \quad r=\frac{\Delta t}{\Delta x} \\
\sum_{k=0}^{s} \alpha_{k} u_{k}(t+\Delta t)=0, \quad \sum_{k=0}^{s} \alpha_{k} \mu^{k}=(1-\mu)^{s} .
\end{gathered}
$$

While this is not of the form (1.4) and $u_{j}(\Delta t)$ must be specified as well as $u_{j}(0)$, our methods still apply.

The operator $S$ which corresponds to this scheme has a generalized eigenvalue at $z=-1$. To see this we look at the roots $\mu$ of $z-z^{-1}=\operatorname{ar}\left(\mu-\mu^{-1}\right)$. The root 
$\mu$ which is inside the unit circle for $|z|>1$ is given by

$$
\mu_{1}=\frac{z^{2}-1-\sqrt{\left(z^{2}-1\right)^{2}+a^{2} r^{2} z^{2}}}{a r z} .
$$

Now for positive $a$, we see that as $z \rightarrow-1$ we have $\mu_{1} \rightarrow 1$; and therefore, $z=-1$ is a generalized eigenvalue of $S$.

If we let $u_{j}(z)=\mu_{1}^{j}(z)$, then proceeding as before, we see that as $z \rightarrow-1$, $\left\|(S-z)^{-1}\right\|$ grows too rapidly for stability.

Thus, for this nondissipative scheme approximating $u_{t}=a u_{x}$, extrapolating at the boundary even with $a>0$ gives an unstable scheme.

6. The General Case. Let us now consider the general explicit difference approximation to (1.1), (1.2), (1.3):

$$
\begin{gathered}
u_{j}(t+\Delta t)=\sum_{k=-r}^{p} C_{k} u_{j+k}(t), \\
u_{j}(0)=f_{j} \\
u_{j}(t+\Delta t)+\sum_{k=1}^{s} \alpha_{j k} u(t+\Delta t)=0, \quad j=-r+1, \ldots, 0 .
\end{gathered}
$$

The scalar product in $H$ now becomes

$$
(w, v)=\left(\sum_{j=-r+1}^{\infty} w_{j} \cdot v_{j}\right) \Delta x
$$

and the operator $S$ is now defined by

$$
\begin{gathered}
(S u)_{j}=\sum_{k=-r}^{p} C_{k} u_{j+k}, \quad j=1,2, \ldots, \\
(S u)_{j}=-\sum_{k=1}^{s} \alpha_{j k}(S u)_{k}, \quad j=-r+1, \ldots, 0 .
\end{gathered}
$$

The general solution to the eigenvalue problem for $|z|>1$ is $u_{j}=u_{j}(z)=$ $\Sigma_{\left|\mu_{i}\right|<1} \sigma_{i} \mu_{i}^{j} P_{i}(j)$, where $\mu_{j}$ are the roots of

$$
\operatorname{Det}\left(\sum_{k=-r}^{p} C_{k} \mu^{k}-z I\right)=0
$$

and $P_{i}(j)$ are polynomials in $j$ with vector coefficients; the order of $P_{i}(j)$ is one less than the multiplicity of the corresponding $\mu_{i}$ (see Kreiss [3]).

If the $\mu_{i}$ are distinct, this becomes

$$
u_{j}=\sum_{\left|\mu_{i}\right|<1} \sigma_{i} \mu_{i}^{j} \phi_{i}
$$


where

$$
\sum_{k=-r}^{p} C_{k} \mu_{i}^{k} \phi_{i}=z_{i} \phi_{i}
$$

In this case for $|z|>1$ there are $r n$ values of $\mu$ with $|\mu|<1$ and $p n$ values of $\mu$ with $|\mu|>1$. The proof is the same as that of Lemma 1(a). As in (b) of Lemma 1 , for each eigenvector $\phi_{i}$ in (6.6), there is exactly one value of $\mu$ which approaches 1 as $z \rightarrow 1$; for $i=1, \ldots, m$ that value of $\mu$ approaches 1 from inside the unit circle; if $i=m+1, \ldots, n$, that value of $\mu$ approaches 1 from outside the unit circle.

Therefore, in the case where $|z|>1$ the roots are distinct and we can write the general solution to the eigenvalue problem

$$
u_{j}=\sum_{i=1}^{r n} \sigma_{i} \mu_{i}^{j} \phi_{i}
$$

In order for $S$ to have a generalized eigenvalue at $z=1$ there must exist $\sigma_{1}$, $\ldots, \sigma_{r n}$ not all zero such that

$$
\sum_{i=1}^{r n} \sigma_{i}\left[\mu_{i}^{j}(1)+\sum_{k=1}^{s} \alpha_{j k} \mu_{i}^{k}(1)\right] \phi_{i}=0, \quad j=-r+1, \ldots, 0 .
$$

In general we know very little about the roots of (6.6) except what we have stated above. We can prove a necessary condition for stability:

For all $w \in E$ (the negative eigenspace of $A$ ) we must have:

$$
\left(I+\sum_{k=1}^{s} \alpha_{j k}\right) w \neq 0 \text { for some } j=r+1, \ldots, 0
$$

If for some $w \in E,\left(I+\Sigma_{k=1}^{s} \alpha_{j k}\right) w=0$ for all $j=r+1, \ldots, 0$, then we can show that the scheme is unstable exactly as we did in the case $r=p=1$.

If we solve explicitly for the resolvent $(S-z)^{-1}$ for $|z|>1$ in an attempt to prove instability when $z=1$ is an eigenvalue of $S$ but we have eliminated from the boundary space the negative eigenvectors of $A$, we run into problems.

Because of the fact that it is now possible for some of the roots $\mu_{i}(z)$ of (6.6) which lie inside the unit circle to coalesce at $z=1$, the boundary matrix (a generalization of (4.3)) may no longer have a factor of $z-1$, but only of $(z-1)^{1 / q}$ where $q$ is the multiplicity of some root $\mu_{i}(1)$ of (6.6) (see Kreiss [3] ).

Appendix 1. Proof That $T(z) M T^{-1}(z)=\left[\begin{array}{cc}M_{1} & 0 \\ 0 & M_{2}\end{array}\right]$. We shall prove that

$$
M T^{-1}=T^{-1}\left[\begin{array}{cc}
M_{1} & 0 \\
0 & M_{2}
\end{array}\right]=\left[\begin{array}{cc}
M_{1} & M_{2} \\
I & I
\end{array}\right]\left[\begin{array}{cc}
M_{1} & 0 \\
0 & M_{2}
\end{array}\right]=\left[\begin{array}{ll}
M_{1}^{2} & M_{2}^{2} \\
M_{1} & M_{2}
\end{array}\right] .
$$

Recall $Q$ is the matrix whose columns are the eigenvectors of $A$. Then 


$$
\begin{aligned}
M T^{-1} & =\left[\begin{array}{cc}
-c_{1}^{-1}\left(c_{0}-z\right) & -c_{1}^{-1} c_{-1} \\
I & 0
\end{array}\right]\left[\begin{array}{cc}
M_{1} & M_{2} \\
I & I
\end{array}\right] \\
& =\left[\begin{array}{cc}
-c_{1}^{-1}\left(c_{0}-z\right) M_{1}-c_{1}^{-1} c_{-1} & -c_{1}^{-1}\left(c_{0}-z\right) M_{2}-c_{1}^{-1} c_{-1} \\
M_{1} & M_{2}
\end{array}\right] .
\end{aligned}
$$

We need only show $-c_{1}^{-1}\left(c_{0}-z\right) M_{1}-c_{1}^{-1} c_{-1}=M_{1}^{2}$ and $-c_{1}^{-1}\left(c_{0}-z\right) M_{2}-c_{1}^{-1} c_{-1}$ $=M_{2}^{2}$. Since $\left\{\phi_{i} \mid i=1, \ldots, n\right\}$ is a basis for $C^{n}$, it is sufficient to show that the two matrices operating on any basis vector give the same vector.

Then for $i=1, \ldots, n$,

$$
\begin{aligned}
-c_{1}^{-1}\left(c_{0}\right. & -z) M_{1} \phi_{i}-c_{1}^{-1} c_{-1} \phi_{i} \\
& =-c_{1}^{-1}\left(c_{0}-z\right) \mu_{i 1} \phi_{i}-c_{1}^{-1} c_{-1} \phi_{i} \\
& =-\mu_{i 1} c_{1}^{-1}\left(\left(c_{0}-z\right)+c_{-1} \mu_{i 1}^{-1}\right) \phi_{i} \\
& =\mu_{i 1}^{2} \phi_{i}=M_{1}^{2} \phi_{i} .
\end{aligned}
$$

The same argument works for $M_{2}$.

Appendix 2. Proof of Lemma 6.

$$
\begin{aligned}
C(z) & =\sum_{k=0}^{s} \alpha_{k} M_{1}^{k+1}=\sum_{k=0}^{s} \alpha_{k} Q\left[\begin{array}{ccc}
\mu_{11}^{k+1} & 0 & 0 \\
0 & \mu_{n 1}^{k+1} & Q^{-1}
\end{array}\right] \\
& =\sum_{k=0}^{s} \alpha_{k}\left[\mu_{11}^{k+1} \phi_{1} \cdots \mu_{n 1}^{k+1} \phi_{n}\right] Q^{-1} \\
\operatorname{det} C(z) & =\operatorname{Det}\left[\begin{array}{c}
p_{11}\left(\mu_{11}\right) \cdots \\
\vdots \\
p_{n 1}\left(\mu_{11}\right) \cdots \\
\vdots \\
p_{n n}\left(\mu_{n 1}\right)
\end{array}\right] \operatorname{Det} Q^{-1}
\end{aligned}
$$

where $p_{k i}\left(\mu_{i 1}\right)$ are polynomials of degree $\geqslant 1$ in $\mu_{i 1}(z)$ and Det $Q^{-1} \neq 0$.

Now $S$ has a generalized eigenvalue at $z=1$ implies that $\operatorname{det} C(1)=0$, which implies that the determinant of the first matrix above is zero at $z=1$.

If we write

$$
\mu_{i 1}(z)=\mu_{i 1}(1)+(z-1) f_{i}(z), \quad f_{i}(1) \neq 0,
$$

by the Corollary to Lemma 1 , then

$$
\operatorname{Det}\left[\begin{array}{ccc}
p_{11}\left(\mu_{11}\right) & \cdots & p_{i n}\left(\mu_{n 1}\right) \\
\vdots & & \vdots \\
p_{n 1}\left(\mu_{11}\right) & \cdots & p_{n n}\left(\mu_{n 1}\right)
\end{array}\right]=\operatorname{Det}\left[\begin{array}{c}
p_{11}\left(\mu_{11}(1)\right) \cdots p_{i n}\left(\mu_{n 1}(1)\right) \\
\vdots \\
p_{n 1}\left(\mu_{n 1}(1)\right) \cdots p_{n n}\left(\mu_{n n}(1)\right)
\end{array}\right]+(z-1) f(z)
$$

where $|f(1)| \leqslant k$. 
Department of Mathematics

C. W. Post College

Greenvale, New York 11548

1. S. K. GODUNOV \& V. S. RYABENKI, Theory of Difference Schemes, An Introduction, North-Holland, Amsterdam, 1964.

2. REUBEN HERSH, "Mixed problems in several variables," J. Math. Mech., v. 12, 1963.

3. H. O. KREISS, "Stability theory for difference approximations of mixed initial boundary value problems. I," Math. Comp., v. 22, 1968, pp. 703-714.

4. H. O. KREISS \& J. OLIGER, Methods for the Approximate Solution of Time Dependent Problems, GARP Publications Series no. 10, Feb. 1973.

5. PETER D. LAX \& BURTON WENDROFF, "Difference schemes for hyperbolic equations," Comm. Pure Appl. Math., v. 17, 1964.

6. S. J. OSHER, "Systems of difference equations with general homogeneous boundary conditions," Trans. Amer. Math. Soc., v. 137, 1969, pp. 177-201.

7. S. J. OSHER, "On systems of difference equations with wrong boundary values," Math. Comp., v. 23, 1969, pp. 567-572.

8. S. J. OSHER, "On certain Toeplitz operators in two variables," Pacific J. Math., v. 34, 1970, pp. 123-129. 